\title{
Contrats et modes de coordination en agriculture
}

Contracts and coordination schemes in agriculture

Zohra Bouamra-Mechemache, Sabine Duvaleix-Tréguer et Aude Ridier

\section{(2) OpenEdition}

\section{Journals}

Édition électronique

URL : http://journals.openedition.org/economierurale/4545

DOI : $10.4000 /$ economierurale. 4545

ISSN : 2105-2581

Éditeur

Société Française d'Économie Rurale (SFER)

Édition imprimée

Date de publication : 15 février 2015

Pagination : 7-28

ISSN : 0013-0559

Référence électronique

Zohra Bouamra-Mechemache, Sabine Duvaleix-Tréguer et Aude Ridier, « Contrats et modes de coordination en agriculture », Économie rurale [En ligne], 345 | Janvier-février 2015, mis en ligne le 15 février 2017, consulté le 19 avril 2019. URL : http://journals.openedition.org/economierurale/4545 ; DOI : 10.4000/economierurale.4545 


\title{
Contrats et modes de coordination en agriculture
}

\author{
Zohra BOUAMRA-MECHEMACHE • TSE Inra Gremaq, Toulouse \\ Sabine DUVALEIX-TRÉGUER • Agrocampus Ouest, UMR 1302 Smart, Rennes \\ Aude RIDIER • Agrocampus Ouest, UMR 1302 Smart, Rennes
}

Cet article cherche à identifier et examiner les modes de coordination et de contractualisation mis en place dans les filières agroalimentaires, en fonction des types de production et des contextes économiques. Les auteures proposent une revue de littérature internationale centrée sur les contrats qui lient les agriculteurs aux entreprises de l'aval des filières. Cette revue examine comment le développement de contrats ou de formes d'organisation alternatives au marché spot répond à des objectifs de performance, d'assurance contre les risques et d'exercice de pouvoir/contrepouvoir de marché. Les auteures étudient le positionnement des organisations de producteurs et des coopératives qui sont un échelon clé dans les filières, à la fois pour organiser les agriculteurs (coordination horizontale) et coordonner l'amont et l'aval de la filière en incluant les entreprises de transformation et de distribution (coordination verticale).

MOTS-CLÉS : contrats agricoles, organisations de producteurs, coordination, risque, efficacité, pouvoir de marché

\section{Contracts and coordination schemes in agriculture}

This article aims at identifying and analyzing coordination and contracting schemes in food supply chains. We provide a literature review related to the contracts between farmers and processing firms. We examine how the development of contracts can be used as an alternative to spot market in order to meet several objectives: performance, risk-management and countervailing market power. We study the role played by producer organizations to improve farmers' horizontal coordination and enhance vertical coordination between the upstream and downstream stages of the industry. (JEL: L22, Q13).

KEYWORDS: agricultural contracts, producers organizations, vertical coordination, risks, efficiency, market power

U ne multitude d'arrangements contractuels existent selon les pays européens et selon les secteurs d'activités agricoles (Ménard et Klein, 2004), avec une gradation de l'absence totale d'engagement à un contrat écrit clairement formalisé. Les acteurs des filières ont recours à la contractualisation pour répondre à des objectifs divers comme la gestion de l'offre, la traçabilité des produits, la garantie d'une qualité ou encore la réduction de coûts de transport (Bogetoft et Olesen, 2002). L'adoption de contrats en agriculture dépend également des caractéristiques et des motivations des agriculteurs (Roe et al., 2004 ; Katchova et Miranda, 2004 ; Zheng et al., 2008). Le recours à des services de conseils et la spécialisation des exploitations en favorisent notamment l'adoption (Katchova et Miranda, 2004). De même que l'entreprise industrielle qui propose un contrat mise ainsi sur le rôle de la confiance entre parties prenantes. Cependant, certaines caractéristiques telles que l'éducation, la taille ou encore l'endettement n'influencent pas toujours significativement l'adoption de contrats. Roe et al. (2004) identifient les coopératives agricoles comme partenaires privilégiés par les agriculteurs. Mais force est de constater que les contrats sont le 
plus souvent mis en place par les industriels (Jaenicke et al., 2007) qui peuvent y trouver un intérêt pour améliorer la performance de leurs entreprises. Les contrats agricoles comportent par ailleurs d'autres spécificités propres à la production agricole. En effet, celle-ci est soumise à des cycles de production longs, souvent de plusieurs mois (céréales, viandes porcines, de volaille), voire de plusieurs années (vins, certains fruits, bovins, etc.). De plus, les biens agricoles sont périssables et donc souvent difficilement stockables. Enfin, les quantités produites sont fluctuantes en raison des aléas climatiques et des risques d'épizootie. Ces spécificités impliquent des modes de coordination propres ou adaptés au monde agricole et agroalimentaire.

Les changements d'organisation dans les filières agroalimentaires ont été accompagnés par un recours plus fort à la contractualisation. Par exemple, le recours aux contrats de production s'est développé en aviculture (États-Unis, Brésil, Union européenne). Aux États-Unis, la production sous contrat ${ }^{1}$ a augmenté de $28 \%$ en 1991 à 38,5 \% en 2008 (MacDonald et al., 2004 ; MacDonald et Korb, 2011). Les produits animaux comptent pour $60 \%$ des contrats de production. Les contrats concernent $90 \%$ de la volaille, $70 \%$ du porc, $55 \%$ du lait, mais seulement $30 \%$ des bovins. Ceux sur les cultures (contrats commerciaux principalement) portent peu sur les grandes cultures (maïs, soja ou blé), même s'ils sont en augmentation, alors que ces cultures représentent une part majeure de la production agricole. En revanche, les contrats sont plus développés pour les

\footnotetext{
1. L'USDA à travers son enquête ARMS (Agricultural Resource Management Survey) évalue régulièrement la production agricole américaine sous contrats. Les contrats sont scindés en deux catégories, les contrats de production et les contrats de commercialisation. Les contrats constituent une forme organisationnelle intermédiaire entre le recours au marché spot et l'intégration verticale.
}

productions végétales où le volume de production est faible (betterave à sucre, tabac et cacahuète).

En France, il n'existe que peu d' information sur l'utilisation des contrats agricoles. Comme aux États-Unis et en Europe, la contractualisation est très répandue en aviculture où plus de $95 \%$ de la production de volailles de chair et plus de $50 \%$ de la production d'œufs de consommation font l'objet de contrats de production ou d'intégration. En revanche, dans d'autres secteurs tels que la filière porcine, les contrats sont peu présents. La commercialisation des porcs charcutiers se fait à travers les organisations de producteurs. Ainsi, en 2009, $93 \%$ des porcs sont commercialisés par 56 Organisations de producteurs (OPs) (Roguet et Rieu, 2011). Dans la filière viande bovine, seuls $20 \%$ des bovins sont commercialisés par les OPs et, selon Coop de France, il n'existe aucun contrat d'intégration.

Dans cet article, nous analysons en quoi ces modes de coordination (contrats, OPs) peuvent répondre aux défis des filières agricoles. Ces défis sont de trois ordres.

- Premièrement, l'évolution de la Politique agricole commune (PAC) a modifié en profondeur le fonctionnement des marchés des matières premières agricoles. Avec le passage d'un soutien des prix agricoles à un système d'aides découplées conditionnelles au respect d'exigences environnementales et de santé, et avec l'ouverture plus forte des marchés domestiques européens au commerce international, les agriculteurs sont aujourd'hui face à une volatilité plus forte des prix des intrants et des produits agricoles. Cette volatilité des prix a pour conséquence d'accentuer la variabilité du revenu.

- Deuxièmement, les produits agroalimentaires sont de plus en plus différenciés horizontalement et verticalement pour répondre aux attentes des consommateurs 
en termes de variété et de qualité des produits. À cela s'ajoutent des exigences en matière de gestion de la sécurité alimentaire le long de la chaîne de production, ce qui renforce le besoin de coordination sur le secteur amont agricole.

- Troisièmement, ces évolutions clés du secteur agricole ont exacerbé l'asymétrie des pouvoirs de négociation entre les différents maillons des filières et ont renforcé le pouvoir d'oligopsone des secteurs de la transformation et de la distribution (OECD, 2006). L'exercice du pouvoir de marché par l'aval de la filière est possible, car l'amont agricole est caractérisé par la présence de nombreuses exploitations agricoles alors que les autres secteurs de la chaîne agroalimentaire (y compris la grande distribution) sont plus concentrés. En France, en 2010, la production de la matière première agricole est très atomisée (490 000 exploitations agricoles) au regard de l'activité de transformation (13 513 entreprises agroalimentaires hors artisanat commercial). De plus, cinq centrales d'achat réalisaient $95 \%$ du chiffre d'affaires au début des années 2000 (Allain et Chambolle, 2003). En Allemagne, les cinq compagnies leaders dans le secteur de la distribution alimentaire réalisent $62,5 \%$ de parts de marché (Herrman et al., 2009). Cette tendance à la concentration du secteur de la grande distribution alimentaire a eu des répercussions sur le secteur de la transformation alimentaire. En particulier, elle a accru la pression sur les prix qui s'est répercutée en amont de la filière.

Ainsi, pour améliorer la performance globale des filières agroalimentaires, encourager l'adoption de stratégies de gestion des risques, ou encore rééquilibrer les rapports de force au sein des filières agricoles et limiter l'exercice du pouvoir de marché du secteur aval, les pouvoirs publics (avec la Loi de modernisation de l'agriculture et de la pêche [LMAP] du 27 juillet 2010 et le règlement européen $n^{\circ} 1305 / 2013$ du 17 décembre 2013 relatif au soutien au développement rural) mettent l'accent sur la coordination verticale dans les filières agricoles et sur le rôle des contrats et des organisations professionnelles et interprofessionnelles ${ }^{2}$, notamment dans les filières lait et viande bovine. En France, la LMAP prévoit notamment dans son volet consacré à " l'amélioration de la compétitivité de l'agriculture » la formalisation de contrats écrits entre agriculteur et acheteur de produits agricoles, ainsi que la possibilité de rendre obligatoire la proposition de contrat soit par extension d'accords interprofessionnels soit sur simplet décret ${ }^{3}$.

L'objectif de cet article est d'identifier et examiner les objectifs économiques visés par les différents types de coordination et de contractualisation mis en place dans les filières agroalimentaires, en fonction des types de production et des contextes économiques. Nous nous concentrerons sur les contrats qui lient les agriculteurs aux entreprises de l'aval des filières. Pour cela, nous examinerons, par une revue de la littérature internationale, comment le développement de contrats ou de formes d'organisation alternatives au marché spot impliquant les agriculteurs peut effectivement répondre à des objectifs de performance, d'assurance contre les risques et d'exercice de pouvoir/contre-pouvoir de marché. Les organisations de producteurs et les coopératives sont un échelon clé dans les filières, à la fois pour organiser les agriculteurs en amont de la filière

2. L'Autorité de la Concurrence a été saisie pour vérifier le respect du droit de la concurrence de ces contrats (avis 08-A-07, 09-A-48 et 10-A-28) et des accords interprofessionnels d'organisation des filières (avis 11-A-03, 11-A-12 et 11-A-14).

3. De tels décrets existent pour les fruits, légumes frais, le lait de vache et un accord professionnel a été rendu applicable dans le secteur de la viande ovine (Danel et al., 2012). 
(coordination horizontale) et coordonner l'amont et l'aval de la filière en incluant les entreprises de transformation et de distribution (coordination verticale). Nous présenterons donc plus précisément leur rôle pour répondre à ces différents objectifs.

L'article est organisé comme suit. Dans un premier temps, nous expliquerons comment les contrats jouent sur la performance des filières en détaillant leurs effets sur l'efficacité des exploitations agricoles et sur l'amélioration de la qualité fournie sur les marchés agricoles et alimentaires. Puis, nous préciserons le rôle des formes de contractualisation dans la gestion des risques liés au marché, à la production et à la demande. Ensuite, nous présenterons en quoi les contrats permettent aux acteurs d'exercer leur pouvoir de marché. Enfin, nous nous intéresserons plus particulièrement au rôle des organisations de producteurs avant de conclure.

\section{Contrats et amélioration de la performance des filières}

\section{Contrats et efficacité}

Le contrat est un outil de coordination pouvant permettre d'améliorer l'efficacité d'une chaîne d'approvisionnement. Un contrat permet en premier lieu de mieux exploiter les économies d'échelle (Melhim et Shumway, 2013 ; Morrison Paul et al., 2004 ; Zheng et Vukina, 2007). Il permet également de réduire les coûts de transaction liés au processus d'échange de biens ou de services (Vukina et Leegomonchai, 2006 ; Wu et Roe, 2007). Il existe en effet un lien étroit entre l'agrandissement des structures agricoles et l'adoption de contrats. Les grandes exploitations qui ont adopté des contrats de production génèrent plus d'efficacité technique et d'échelle même si l'effet isolé des contrats est limité (Morrison Paul et al., 2004). Cet effet a été retrouvé pour des agriculteurs adoptant des contrats de commercialisation dans les filières blé, maïs et lait (Melhim et Shumway, 2013). Les exploitations agricoles qui ont développé des contrats de commercialisation variés présentent des économies d'échelle spécifiques à chaque produit. Ainsi, l'agrandissement des structures agricoles est plus rapide en présence de contrats de commercialisation sur le blé et le maïs qu'en production laitière où l'adoption de contrats est indépendante de l'évolution des structures.

De la même manière qu'une entreprise cherche à exploiter les économies d'échelle, elle vise à réduire ses coûts de transaction en privilégiant une forme organisationnelle (recours au marché, contrats, intégration verticale). Quatre types de coûts de transaction sont classiquement liés aux contrats. À la date de signature du contrat, il peut être, 1) soit trop coûteux de recenser toutes les situations futures possibles, 2) soit impossible de prévoir l'ensemble de ces événements. Après la signature du contrat, 3) des coûts de contrôle, voire 4) des coûts juridiques apparaissent afin d'assurer le respect des engagements. Selon la situation de l'entreprise et le fonctionnement des marchés, une entreprise agricole préférera, pour l'échange de ses produits, recourir au marché, développer des relations contractuelles ou encore intégrer verticalement une partie de sa production (Williamson, 1975).

Aujourd'hui, l'accent est mis sur la formalisation des contrats. Cependant si la rédaction d'un contrat formel représente un point de départ dans la relation entre deux partenaires, les contrats incluent souvent un champ plus large d'informations subjectives qui inciteront les entreprises à respecter elles-mêmes leur engagement afin de maintenir leurs relations futures (Baker et al., 2002 ; Levin, 2003). Les contrats informels émergeront plus facilement dès lors que le nombre des transactions est élevé, que la fréquence des événements imprévus est relativement faible et que l'entreprise dépend étroitement de sa 
réputation. En agriculture, certaines règles peuvent être établies de manière plus ou moins informelle sur les délais de livraison, les engagements de qualité ou encore la fréquence de renouvellement du contrat. Par conséquent, il peut être difficile pour un tiers d'évaluer le respect de l'engagement du contrat.

\section{Contrats et mécanismes d'incitation à la performance}

En général, les comportements de chacune des parties (agriculteurs et industriels) dans leur activité économique jouent un rôle sur l'échange et sur la performance globale de la chaîne d'approvisionnement. Or les choix d'une des parties ne sont pas forcément observés par l'autre partie contractante. Dans une relation pure de marché, ces situations peuvent conduire à certaines inefficacités. Par comparaison à une relation de marché, le contrat peut résoudre en partie ces inefficacités en donnant des incitations pour les parties à se comporter favorablement. Le contrat peut ainsi définir une conduite d'élevage ou un itinéraire technique de production précis que l'agriculteur s'engage à suivre. L'industriel peut désigner des conseillers techniques pour suivre régulièrement les producteurs. Dans cette section, nous analysons plus spécifiquement trois mécanismes d'incitation à la performance. Le premier porte sur l'incitation à la performance prix/quantité. Le deuxième sur l'incitation à la qualité. En effet, la qualité des facteurs (potentiel génétique animal, mélange d'aliments) constitue un élément déterminant de la qualité du produit final. Le troisième mécanisme d'incitation par les contrats est celui permettant aux agriculteurs de développer des innovations.

\section{Contrats et performance prix/quantité}

Les contrats peuvent permettre d'améliorer la performance quantitative de la production en incitant les agriculteurs à une meilleure utilisation des facteurs et en les rémunérant suivant un indice de performance (par exemple le rapport poids vif sur quantité d'aliments). Cependant, tous les contrats, à travers la mise en place d'un schéma de rémunération propre, n'atteignent pas le même degré d'efficacité. Zheng et Vukina (2007) par exemple comparent des schémas de rémunération basés sur la performance des éleveurs dans les contrats de production en aviculture. Ils montrent que le passage d'une rémunération basée sur le seul classement de la performance individuelle à une rémunération basée sur la performance moyenne du groupe est bénéfique pour le transformateur qui est en mesure d'augmenter la quantité produite. L'industriel est de plus en mesure de baisser le paiement moyen à l'éleveur, ce qui implique un résultat mitigé pour ce dernier en fonction des chocs de productivité qu'il subit.

D'autres facteurs, tels que l'aversion au risque ou la présence d'asymétries d'information, jouent sur l'efficacité de la relation contractuelle. Dubois et Vukina (2004) s'y intéressent dans les contrats de production en élevages porcins. L'asymétrie d'information provoque non seulement des risques de baisse de la performance des élevages, en raison d'une moindre incitation à l'effort, mais aussi des risques sur les choix de l'industriel qui alloue le volume de production de l'éleveur en fonction du degré d'aversion au risque de ce dernier. Ainsi, les éleveurs présentant le plus d'aversion au risque percevront non seulement une rémunération plus faible, mais également moins d'animaux à élever. Goodhue (2000) montre qu'en proposant un contrat, l'industriel peut contrôler les intrants et réduire la rente informationnelle des éleveurs les plus productifs ainsi que faire supporter une partie du risque systémique sur les élevages.

\section{Contrats et incitation à la qualité et à l'innovation}

Les contrats et les modes d'organisation au sein des filières ont une incidence sur le 
développement de standards de qualité et sur les mécanismes d'incitation à la qualité.

Les attentes des consommateurs sur l'alimentation se font plus vives sur les critères de sécurité sanitaire, de santé et d'environnement, obligeant les pouvoirs publics à développer des standards de qualité minimum sur l'ensemble de la chaîne alimentaire. L'adoption du Paquet Hygiène qui soumet, depuis 2006, l'ensemble des acteurs de la chaîne alimentaire en Europe aux mêmes exigences de traçabilité, d'identification, d'analyse des risques sanitaires et de bonnes pratiques d'hygiène en est un exemple. Les différentes crises sanitaires de la fin des années 1990 et du début des années 2000 ont détérioré la confiance des consommateurs qui sont devenus sensibles et exigeants sur les systèmes de production agricole et les processus de fabrication des produits agroalimentaires, augmentant ainsi la demande en produits certifiés ${ }^{4}$. Les distributeurs ont également créé des référentiels privés (comme les référentiels Bristish Retail Consortium $B R C$, International Food Standard IFS, Global Gap) plus contraignants que les standards de qualité minimum développés par les pouvoirs publics. Ils jouent un rôle de leader pour augmenter la qualité de leurs produits (Hennessy et al., 2001) et prendre un risque minimal sur leur réputation (Fulponi, 2006), même si l'utilisation de ces référentiels n'élimine pas nécessairement tous les risques de sécurité sanitaire dans la chaîne alimentaire 5 . Ces référentiels privés ne sont pas négociés, mais

4. Trienekens et Zuurbier (2008) décrivent et expliquent en détail les différents systèmes de certification obligatoires et volontaires mis en place pour garantir la sécurité sanitaire des produits alimentaires.

5. Comme le montrent Giraud-Héraud et al. (2012), seule une certification élaborée par l'ensemble des distributeurs peut empêcher les risques de passager clandestin de distributeurs en raison de la difficulté à différentier les produits certifiés par leur attribut de sécurité alimentaire. imposés aux fournisseurs de produits agricoles et agroalimentaires. Ils renforcent par conséquent le pouvoir de négociation des acteurs en aval qui peuvent exclure du marché les fournisseurs n'ayant pas adopté le standard. Ces référentiels font donc partie intégrante du contrat entre le distributeur et l'industriel. À l'inverse, lorsque les agriculteurs initient un standard de qualité, ils peuvent le définir de telle sorte à récupérer de la valeur dans la filière (BouamraMechemache et Yu, 2013). D'autres logiques de standardisation existent dans les filières agroalimentaires comme le développement de normes qui sont par nature à caractère consensuelle et collective. Par exemple, la norme NF V 46-007 s'applique à la filière viande pour garantir la traçabilité des produits. Si l'ensemble de ces démarches partage un objectif commun de garantir la sécurité alimentaire des produits, leur élaboration et mise en application sont diverses (Valceschini et Saulais, 2005). Le développement de ces multiples démarches de standardisation soulève la question de leur positionnement dans les relations contractuelles. Complètent-elles les contrats en imposant des critères de qualité à la fabrication des produits alimentaires ? Ou se substituentelles aux relations contractuelles déjà existantes ? La réponse à ces questions n'est pas triviale et nécessite une exploration qui dépasse le champ de ce papier.

Dans le cadre d'une politique de qualité et de sécurité sanitaire des aliments, les contrats ont souvent été utilisés pour s'assurer d'une qualité suffisante de l'offre (Lawrence et al., 2001). Ainsi, les acteurs de la grande distribution ont recours au marché spot pour l'approvisionnement en produits génériques. Par contre, pour leurs marques propres, ils ont recours à des relations contractuelles avec les agriculteurs pour garantir que les produits soient conformes aux standards de production plus restrictifs, notamment dans les secteurs de la viande et des fruits et légumes. 
Ces relations contractuelles peuvent être bénéfiques aux agriculteurs fournissant une production de meilleure qualité (Bazoche et al., 2005).

La coordination verticale à travers la contractualisation favorise ainsi la production de biens de meilleure qualité (Goodhue et al., 2003 ; Goodhue, 2011). Cependant, les contrats écrits spécifient plusieurs autres attributs tels que le système de production ou les primes. La qualité globale d'un produit reflète souvent l'association de plusieurs attributs qui peuvent jouer positivement ou négativement sur le produit. Peu de travaux ont étudié l'effet de primes sur la qualité des produits. Goodhue et al. (2010) examinent cette question sur la production de tomates. Ils mettent en évidence un effet différencié des primes selon le critère de qualité récompensé.

Enfin, les modes de coordination verticale peuvent jouer un rôle déterminant dans le développement d'innovations en limitant le risque d'expropriation (Karantininis et al., 2010). La coordination verticale garantit également la protection de la valeur des droits de propriété liée à l'innovation technologique. Dimitri et al. (2009) expliquent ainsi le passage d'une relation de marché aux contrats en aviculture (Knoeber, 1989). De même, les coopératives agricoles, par les liens étroits qui lient l'amont agricole à la transformation industrielle peuvent faire coïncider les objectifs des deux maillons des filières et favoriser le développement d'innovations. Giannakas et Fulton (2005) et Drivas et Giannakas (2010) montrent ainsi que la présence de coopératives peut améliorer le taux d'innovation tout en réduisant le prix d'achat du bien pour les adhérents.

\section{Contrats, gestion des risques de marché et de production}

Les risques qui entourent l'activité agricole sont de diverses natures (Hardwood et al., 1999). Les sources de risque les plus importantes sont (i) les risques de prix, liés à la difficulté d'anticiper les fluctuations des prix des produits et des intrants, (ii) les risques liés à la demande ou au débouché, lorsque par exemple la production ne trouve pas d'acquéreur à la suite des changements non anticipés des préférences des consommateurs. Enfin (iii) les risques de production ou d'offre liés à la fluctuation du niveau de la production résultant de mauvaises conditions climatiques, des problèmes attachés à l'aliment, au travail ou à l'apparition de maladies affectant les récoltes (Meuwissen et al., 2001 ; Patrick et al., 2007 ; Hardaker et al., 2004) ou encore à des problèmes sanitaires (vache folle, listeria). Trois types de risque de production peuvent être identifiés : le risque de production spécifique à un producteur ; le risque de production commun aux producteurs soumis, par exemple, aux mêmes aléas climatiques et sanitaires ; enfin, lorsque la production ne satisfait pas à des critères sanitaires minima, on parle de risque-qualité.

Le prix de marché du produit est influencé par des facteurs dépendant de l'offre et de la demande. Les agriculteurs sont souvent exposés à des risques de prix et le seront sans doute plus à l'avenir, avec la baisse significative des prix de soutien dont le coût repose sur le contribuable ou le consommateur. Avec l'élimination de cette forme d'assurance fournie par le mécanisme de soutien des prix, le contrat peut devenir un outil majeur d'assurance contre le risque-prix.

La plupart des producteurs ont de l'aversion pour le risque et sont prêts à payer une prime pour s'assurer et réduire leur exposition au risque. Ils peuvent avoir recours à des contrats d'assurance. Cependant, peu de contrats d'assurance privée prennent en charge en France les dommages spécifiquement agricoles, à l'exception, en matière de risque climatique, de l'assurance-grêle 
et multirisque climatique, ceci en raison du caractère systémique des risques de marché et de certains risques sanitaires ou de récolte.

La coordination verticale dans les filières peut aussi permettre au producteur de se couvrir contre différents risques (de prix, de débouché) en offrant un meilleur partage de ces risques (Pietola et Wang, 2000). Inversement, pour s'assurer contre le risque lié à l'offre, une entreprise peut stipuler un contrat qui engage les agriculteurs à lui livrer leur production et qui réciproquement engage l'entreprise à l'acheter. Le producteur est ainsi assuré que sa production trouvera un débouché et l'entreprise contractant avec plusieurs producteurs obtient l'assurance d'un approvisionnement régulier, les différents risques spécifiques des producteurs se compensant en moyenne. Cependant, avec ce type de contrat, le risque commun de sur- ou sousproduction peut rester significatif pour l'entreprise si celle-ci a un approvisionnement local et peu diversifié. Lorsque l'entreprise fait face à une demande aléatoire, si elle s'engage sur une quantité de commande, le risque pour elle est alors soit d'avoir un excédent de production ou soit de ne pouvoir satisfaire la demande lorsque celle-ci est connue. En gérant une demande variable de ses clients, elle subit les variations de quantité qui sont amplifiées en fonction des prévisions effectuées, des pénuries possibles sur l'approvisionnement, des délais d'obtention des produits, du groupement des commandes en lots ou encore des variations de prix (Lee et al., 2004).

Les contrats de commercialisation peuvent être proposés aux agriculteurs dans les pays développés, en complément ou non des contrats de production ( $c f$. encadré). Ils sont d'une grande diversité. Dans le secteur des cultures aux États-Unis, ils confèrent plus de droits de contrôle et de décision aux producteurs que les contrats

\section{Encadré. Les contrats de production}

Dans ce type de contrat, l'agriculteur est rémunéré pour les services qu'il rend. Le donneur d'ordre (l'intégrateur) confie au producteur la mission de produire une quantité répondant à un cahier des charges particulier et s'engage en contrepartie à acheter la production réalisée (Zheng et al., 2008). Deux types de contrats portant sur les quantités existent. Les premiers portent sur un engagement minimum d'achat. Les seconds permettent au client de conserver une part de flexibilité concernant la quantité commandée. Dans l'industrie du porc aux États-Unis, ces types de contrats peuvent porter sur une ou plusieurs étapes de la production (comme les contrats d'engraissement de porcelets). L'accord porte sur deux composantes, la responsabilité de la fourniture des inputs et la détermination de la compensation financière. L'existence de ce contrat prémunit l'éleveur face au risque de fluctuation des prix des aliments, car c'est l'industriel qui fournit la majorité des inputs variables. Dans le secteur des grains, les prescripteurs de ce type de contrat peuvent être des entreprises de semence ou des coopératives pour lesquelles la production de grains doit répondre aux exigences de certains industriels (blé dur par exemple).

de production dans le secteur de l'élevage (Paulson et al., 2010). Ils portent sur le produit échangé et spécifient le prix du bien ou un mécanisme pour le déterminer, le lieu de livraison, la quantité à livrer et le degré d'exposition au risque de marché, et sa durée (à travers les dates de paiement).

Dans les sections suivantes, nous présentons les différentes formes existantes de contrats de commercialisation en agriculture et la façon dont ils prennent en charge le risque-prix (1), puis, nous discutons du rapport entre l'assurance apportée par ces contrats et leur caractère incitatif (2).

\section{Les contrats de gestion du risque prix}

En matière de commercialisation des grains, on peut distinguer plusieurs types de 
contrats de commercialisation : celui à prix de vente moyen (pool contract), le contrat à prix ferme avant (forward contract) et au moment de la récolte (spot), le contrat à prix indexé (Reynaud et Ricome, 2010).

Les contrats à prix de vente moyen (pool contracts)

Ces contrats sont très développés en France $^{6}$, on les retrouve aussi aux ÉtatsUnis dans le secteur du coton. Signés avec l'entreprise de collecte, ces contrats permettent au producteur d'obtenir un prix garanti dès la récolte et de soulager ainsi sa trésorerie, avec la possibilité de profiter des éventuelles hausses de prix ultérieures. Pour l'entreprise de collecte, ces contrats permettent, en commercialisant régulièrement une proportion constante de volumes collectés, de mutualiser entre les adhérents, le risque de variabilité du prix sur une campagne. Dans ce type de contrat, la commercialisation est entièrement déléguée à l'entreprise de collecte. D'autres types de contrats de commercialisation se développent en France depuis le début des années 2000 dans les filières-grains, probablement en raison d'une volatilité plus forte des prix. Ces contrats ne sont pas basés sur un prix de vente moyen, ce qui permet à l'agriculteur de se réapproprier la responsabilité des choix commerciaux (Coop de France, 2010).

\section{Le contrat à prix ferme}

Le contrat à prix ferme à la récolte (spot) est conclu pour un prix représentatif du marché physique. Le contrat à prix ferme avant récolte (forward contract) est conclu pour une quantité, une qualité et une période de livraison. Ce type de contrat permet au producteur de réaliser une opération de couverture du risque de fluctuation

6. Les contrats à acompte et complément de prix représentent le système " historique » dans les coopératives céréalières françaises, ils sont les plus répandus (Coop de France). du prix et de disposer d'une certaine visibilité sur les futures rentrées de liquidités. Il lui permet aussi d'être payé très rapidement après la livraison de la marchandise. Cependant, ce type de contrat expose à de nouveaux risques. Le premier est le risque de non-conformité si la livraison ne permet pas de tenir les engagements en quantité et qualité et le second est le risque de contrepartie si l'une des deux parties, profitant d'une baisse ou d'une hausse des cours, tente de se rétracter de ses engagements. C'est pour limiter les risques de débouché et de fluctuation des prix que les marchés à terme se sont développés (Carter, 2013). Mais ce sont aujourd'hui et pour diverses raisons les acteurs des filières (coopératives, industriels) plutôt que les agriculteurs eux-mêmes qui les utilisent. Dans la filière laitière aux États Unis, ces types de contrats disponibles dès la fin des années 1990 restent peu développés malgré l'augmentation de la volatilité des prix, ceci en grande partie en raison du risque de base (Wolf, 2012).

\section{Le contrat à prix indexé}

Dans ce type de contrat, tous les éléments de la transaction sont arrêtés (quantité, qualité, date de livraison) sauf le prix, pour lequel seule la modalité de fixation est spécifiée (et non la valeur). Elle peut se faire sur la base d'un indice de prix ou d'un prix minimum. Là encore, c'est le producteur qui se réapproprie l'activité de commercialisation.

Le lien entre l'utilisation des contrats à terme (forward contracts) et l'attitude par rapport au risque a été testé auprès de 440 éleveurs de porcs aux Pays-Bas (Pennings et Leuthold, 2000 ; Pennings et Garcia, 2001). Ils montrent que l'attitude par rapport au risque joue un rôle sur l'adoption des contrats pour certaines catégories d'éleveurs où les variables financières pèsent aussi, comme le taux d'endettement. De même, le niveau d'aversion au risque influence l'adoption des 
contrats de production (Dubois et Vukina, 2004). Les utilisateurs de contrats de commercialisation ou du marché-spot sont moins averses au risque que les utilisateurs de contrats de production dans l'industrie du porc aux États-Unis, car l'exposition au risque est moins forte dans un contrat de production que dans un contrat de commercialisation (Zheng et al., 2008). Ainsi, les contrats à terme permettent aux agriculteurs de gérer eux-mêmes le risque sur les prix selon leur objectif de revenu et le risque qu'ils sont prêts à prendre. De tels contrats de gestion du risque-prix n'existent pas dans les filières de production animale en France, ils sont cependant largement développés dans les productions céréalières. Ces types de contrats ont été développés aux États-Unis dans le secteur laitier depuis le début des années 1990. Le changement dans les politiques agricoles a entraîné de plus grandes variations de prix et par conséquent, une augmentation des risques pour les agriculteurs et les industriels. Les coopératives laitières américaines ont donc lancé de nouveaux contrats pour répondre aux besoins émergents de gestion du risque-prix de leurs adhérents. Elles leur ont proposé des contrats à prix minimum et des contrats à prix déterminé à l'avance - la durée de ces contrats peut varier d'un mois à une année. Le plus souvent, les coopératives limitent ces contrats à une certaine proportion de la production estimée de chacun de leurs adhérents. Pour gérer ces contrats avec un minimum de risque, les coopératives peuvent prendre des positions sur les marchés à terme, tout comme pour les contrats à prix indexé en céréales. Sans couverture sur les marchés à terme, les contrats de gestion du risque-prix affectent à la fois le niveau et la variabilité du prix moyen perçu par les adhérents d'une coopérative laitière qui restent sous le contrat de base. Le choix de proposer de tels contrats dépend des réserves financières de la coopérative, de sa position sur les marchés d'aval ainsi que de l'hétérogénéité de ses adhérents (Hovelaque et al., 2009).

\section{Mécanisme d'assurance ou mécanisme d'incitation ?}

Le recours aux contrats pour résoudre des problèmes de risque peut également avoir des répercussions sur les incitations à la performance. Un résultat fondamental de l'économie des contrats est qu'il existe un arbitrage entre incitations et assurance (Salanié, 2005). Ceci signifie que plus un contrat permet à un agent de s'assurer contre le risque, moins il fournit d'incitations à ce même agent. Les contrats observés dans l'agriculture ne font pas exception (Hueth et al., 1999).

Si la rémunération de l'agriculteur ne dépend pas de ses performances (salariat par exemple dans le cadre d'un contrat de production), l'agriculteur est complètement assuré contre les risques de production par la partie contractante, mais ses performances peuvent en être affectées. Il est donc face à un arbitrage. Il peut s'assurer face au risque de production en acceptant le contrat proposé par l'industriel ou vendre sa production au prix du marché. Dans ce cas, sa rémunération dépend entièrement de ses performances, mais il subit complètement les risques communs et spécifiques de production. Ainsi, le contrat doit permettre un équilibre entre assurance contre les risques et incitations à produire.

Le même type de dilemme peut exister pour l'entreprise aval. Lorsque le risque de production est complètement supporté par l'agriculteur, l'intégrateur dans le cas du contrat de production peut ne pas être incité à fournir les facteurs de production les plus favorables pour l'agriculteur. Par exemple, dans le cas des contrats de production en aviculture (Magdeleine, 2012), il peut avoir intérêt à fournir des aliments et des poussins de moins bonne qualité en termes de composition de l'aliment ou d'âge des poules pondeuses puisque ce n'est pas lui 
qui supporte les risques (Leegomonchai et Vukina, 2005). Ainsi, plus le collecteur supporte les risques de production, plus ses incitations à fournir des facteurs de production de qualité augmentent.

\section{Contrats et pouvoir de marché}

Si les contrats peuvent être utilisés pour résoudre des défaillances économiques liées aux risques et à la performance, la mise en place de contrats (y compris l'intégration verticale) est également un outil permettant aux entreprises d'exercer leur pouvoir de marché sur les producteurs. Pour maintenir leur pouvoir de négociation face à un secteur de la distribution plus concentré, une vague de fusions et d'acquisitions des entreprises du secteur agroalimentaire (Vavra, 2009) a succédé à la vague de fusion de la grande distribution. Il en résulte que les entreprises en aval de la filière exercent potentiellement un pouvoir d'oligopole sur les marchés des produits finis et un pouvoir d'oligopsone sur les marchés d'approvisionnement en matière première sur les marchés agricoles en amont de la filière. Azzam (1997) montre qu'un pouvoir de marché lié à la concentration forte du secteur existe sur l'industrie de la viande bovine américaine. Cependant, il estime que les gains d'efficacité liés aux économies d'échelle sont supérieurs au pouvoir de marché généré par l'augmentation de la concentration du secteur, ce que confirment les travaux de Morrison-Paul (2001). Celle-ci examine également le pouvoir de monopsone des abattoirs et révèle un faible pouvoir de marché des abattoirs sur la matière première agricole.

La production agricole, quant à elle, n'est pas ou peu concentrée. Ainsi, la concentration $(\mathrm{C} 4)^{7}$ est faible dans les secteurs du lait $(0,2 \%)$, de la volaille, des

7. Le C4 représente la part du chiffre d'affaires des quatre premières entreprises sur le chiffre d'affaires total du secteur. œufs, du veau et de la tomate (5\%) aux Pays-Bas (OECD, 2006). En revanche, avec l'émergence d'organisations de producteurs, les producteurs ont la possibilité de regrouper l'offre de produits agricoles, la concentration de la production agricole peut alors devenir plus forte dans certains secteurs comme celui des fruits et légumes (48,3\% dans le cas des tomates cerises par exemple). La mise en place de contrats et l'intégration verticale de l'amont permettent aux acteurs des filières d'exercer leur pouvoir de marché sur les producteurs. Ainsi, les changements d'organisation dans les filières agroalimentaires ont été accompagnés par un recours plus fort à la contractualisation.

La position d'oligopsone des opérateurs peut leur permettre de bénéficier d'un pouvoir de marché vis-à-vis des producteurs et de réduire les prix des intrants agricoles. Cet exercice du pouvoir de marché peut même conduire les agriculteurs à produire à un prix inférieur au coût marginal dans certaines régions, conduisant à une perte d'activité économique et à la dépopulation de certaines zones rurales (Crespi et al., 2012). L'exercice de pouvoir de marché dépend de la présence de barrières à l'entrée, de l'existence d'alternatives possibles sur le marché et de la nature des produits vendus. Des barrières à l'entrée peuvent exister dans le cas où les coûts de transport sont élevés ou si les produits sont périssables et ne peuvent pas être transportés sur une longue distance. Dans ce cas, l'absence de débouchés pour la production agricole renforce le pouvoir de marché des établissements présents sur la zone géographique de production. C'est le cas par exemple de l'industrie avicole aux ÉtatsUnis (Vukina et Leegomonchai, 2006), où les éleveurs ont une opportunité limitée à contractualiser avec d'autres entreprises, car le transport sur longue distance des volailles est impossible. C'est également le cas dans le secteur laitier, car le lait est périssable et doit être livré rapidement 
dans les entreprises de transformation, une fois collecté. Cette caractéristique du marché de la volaille ou du lait confère aux transformateurs un pouvoir d'oligopsone (voir de monopsone) sur les éleveurs dans une zone géographique donnée.

L'exercice de pouvoir de marché à travers les contrats peut prendre plusieurs formes. Lorsqu'ils sont en situation d'oligopsone, les acheteurs peuvent imposer des contrats aux producteurs et spécifier les termes du contrat de telle sorte qu'ils soient à leur avantage (voir Mac Donald, 2006).

\section{Contrats et risque de hold-up}

Dans le cas où la production demande des investissements spécifiques, le contrat peut lier fortement le producteur à son acheteur, car il ne lui sera pas facile (souvent impossible) de trouver un autre acheteur. C'est le cas par exemple du secteur du lait. Le marché du lait cru est captif, les éleveurs ont peu d'options de sortie (Dedieu et Courleux, 2009). Il est ainsi difficile aux éleveurs de trouver des opportunités extérieures avantageuses. De plus, les investissements y sont spécifiques et la qualité du produit est hétérogène. Dans ce cas, des relations de long terme entre les éleveurs et leur entreprise de collecte (via des contrats) sont nécessaires.

Lorsqu'il n'existe pas d'options de sortie pour leurs fournisseurs, l'acheteur peut alors être tenté de profiter de cette situation et de payer un prix trop bas qui ne permettra pas de couvrir les coûts d'investissements. En revanche, l'acheteur peut également avoir intérêt à imposer des contrats pour assurer son approvisionnement. En l'absence de contrats, il pourrait être plus difficile pour lui de trouver des producteurs prêts à investir sans un minimum de garantie. Vukina et Leegomonchai (2006) illustrent le problème de hold-up dans le cas de l'industrie avicole. En plus de l'existence d'un pouvoir de marché lié à l'absence d'alternative de débouchés pour les éleveurs, cette industrie se caractérise par la spécificité des actifs de production. Les actifs de production doivent être conformes aux spécificités de production définies par chaque transformateur et mentionnées par contrat. Dans ce cas, les actifs de production n'ont de la valeur que dans le cadre de la relation bilatérale avec le transformateur concerné. Les transformateurs peuvent tirer profit de cette situation pour exercer leur pouvoir de marché sur les éleveurs et récupérer la rente procurée sur l'investissement.

Dans ce cas, l'incitation à investir des éleveurs peut être affectée. Cette situation de hold-up entraîne un investissement sous-optimal par rapport à une situation où l'entreprise intègre verticalement les éleveurs (voir aussi Reimer [2006] dans le secteur du porc). Ainsi, l'analyse empirique de Vukina et Leegomonchai (2006) montre que les investissements spécifiques des éleveurs dépendent systématiquement du pouvoir de marché des entreprises et du degré de spécificité de l'actif. Une augmentation de la spécificité de l'actif entraîne une compensation plus faible des éleveurs que dans la situation où l'intégrateur était en situation de monopsone. De plus, la taille des investissements est positivement reliée au nombre d'intégrateurs qui contractent les services de l'éleveur dans une région donnée. Ce problème de hold-up est également présent dans les relations entre industrie de transformation et grande distribution.

\section{Contrats et limitation de la concurrence}

Les contrats peuvent également être utilisés pour éviter une concurrence trop agressive sur le marché spot et donc agir sur les prix intermédiaires. Par exemple, Xia et Sexton (2004) analysent les contrats mis en place par l'industrie de conditionnement de viande bovine pour l'achat de bovin. Le recours à un contrat spécifiant le prix de 
base au niveau le plus haut sur le marché des bovins peut dissuader une concurrence agressive en facilitant une collusion tacite des opérateurs pour réduire les prix du marché spot. L'analyse de Xia et Sexton repose sur un modèle théorique de concurrence à la Cournot sur un marché d'approvisionnement en situation de duopsone. Dans ce cadre, le recours aux contrats a pour effet de réduire la concurrence et de baisser les prix au niveau de monopsone dans le cas où $50 \%$ de l'offre est sous contrat et où la production de bovins sous contrat ne peut pas s'ajuster à la baisse. En revanche, si l'offre peut s'ajuster, la baisse des prix n'a lieu que si les industriels utilisent un contrat de long terme. Avec un contrat de court terme, le prix d'équilibre est la solution de Cournot (Zhang et Brorsen, 2010) et implique une baisse de prix plus faible qu'à long terme (les prix sont néanmoins inférieurs aux prix de concurrence parfaite). Cependant, les études empiriques ne semblent pas indiquer l'exercice d'un tel pouvoir de marché (Ward et al., 1998 ; Key, 2011). Ces études montrent en effet que le recours aux contrats n'a pas d'effet négatif (ou un effet très faible) sur les prix des bovins aux États-Unis. Seule l'analyse de Crespi et Johns (2005) indique une baisse des prix entre 5 et $10 \%$ liée au pouvoir d'oligopsone. L'absence d'effet prix pose la question de la justification du recours aux contrats par l'industrie de conditionnement. Les contrats peuvent être utilisés pour garantir l'approvisionnement. En effet, ce n'est pas l'intérêt à long terme des transformateurs de limiter les quantités achetées pour affecter les prix d'approvisionnement, car cela entraînerait la sortie de producteurs et la réduction des capacités productives alors que ces capacités sont nécessaires pour maintenir les ressources dans l'industrie (Sexton, 2013).

Pour éviter la concurrence d'une entrée potentielle d'entreprises sur le marché, une entreprise peut également utiliser les contrats pour restreindre l'entrée de concurrent. C'est le cas par exemple pour les entreprises d'emballage des viandes (Ward, 2002). Dans ce secteur, la performance repose sur la possibilité d'exercer des économies d'échelle : l'accès à de grandes quantités d'animaux leur permet de rentabiliser leurs unités de transformation. Si un transformateur réussit à obtenir une grande proportion de l'offre locale à travers l'utilisation de contrats de long terme, il peut diminuer l'offre potentielle pour ses concurrents et augmenter ainsi leurs coûts d'approvisionnement pour se procurer une offre en quantité suffisante. Il peut alors dissuader l'entrée d'un concurrent en augmentant le coût de production auquel il doit faire face. Une fois l'entrée dissuadée, il peut ensuite faire baisser le prix sur le marché spot en limitant les achats de bétail. Cependant, comme discuté dans la section 1, de telles pratiques peuvent également avoir des effets positifs pour la filière et améliorer l'efficacité des filières agricoles. De grands volumes de production sont, en effet, nécessaires pour exploiter des économies d'échelle. Il est ainsi nécessaire pour une entreprise de s'assurer un approvisionnement suffisant avant d'investir dans des équipements de production lourds et intensifs en capital.

\section{Coopératives et organisations de producteurs}

Dans la section précédente, nous avons identifié plusieurs raisons qui peuvent expliquer l'intérêt pour les entreprises de transformation à développer des contrats pouvant aller jusqu'à l'intégration complète de l'activité de production en amont (réduire leurs coûts de transaction, les risques et corriger les distorsions de marché engendrées par l'exercice de leur pouvoir de marché).

Les agriculteurs peuvent également avoir intérêt à intégrer une partie des activités aval et à se coordonner horizontalement pour bénéficier d'économies d'échelle et 
contrebalancer le pouvoir de marché de l'aval (Sexton et Lavoie, 2001). Ainsi, les agriculteurs peuvent se coordonner sous la forme de coopératives ou d'autres formes d'Organisations de producteurs $(\mathrm{OPs})^{8}$. $\mathrm{Ce}$ mode de coordination est répandu en France comme dans l'ensemble de l'Union européenne même s'il existe une grande variation d'un pays membre à l'autre. Ainsi, la part de marché des coopératives agricoles est de $40 \%$ en moyenne dans l'Union européenne et de $55 \%$ en France (Bijman et al., 2012).

\section{OPs et contre-pouvoir de marché}

Le rassemblement des producteurs sous forme de coopérative permet de réduire l'asymétrie dans les pouvoirs de négociation entre les maillons agricole et de transformation, au sein des filières, et renforce ainsi le rôle des coopératives agricoles (Roe et al., 2004 ; Zeuli et King, 2004). En se regroupant dans des OPs, les agriculteurs peuvent en effet changer la nature de la concurrence sur les marchés agroalimentaires. Le passage d'une structure purement concurrentielle à une structure oligopolistique de la production agricole peut conférer un contre-pouvoir de marché aux producteurs. Ainsi, l'analyse des coopératives en Europe, conduite par Bijman et al. (2012), indique que les coopératives agricoles en Europe permettent effectivement aux agriculteurs de capter une partie plus importante de

8. Le ministère de l'Agriculture définit une OP en France comme " une organisation constituée à l'initiative d'un ensemble d'agriculteurs qui se regroupent dans l'objectif de mutualiser leurs moyens afin de rééquilibrer les relations commerciales qu'ils entretiennent avec les acteurs économiques de l'aval de leur filière ». Une société coopérative agricole, une union de coopératives agricoles, une société d'intérêt collectif agricole, une association régie par la loi du $1^{\text {er }}$ juillet 1901, une société commerciale ou un groupement d'intérêt économique peut être reconnu, par arrêté ministériel, organisation de producteurs (OP). la valeur ajoutée des produits créée dans la chaîne de production. D'autres études cependant viennent nuancer l'impact que peuvent avoir les OPs sur le partage de la valeur. En effet, Ahn et Sumner (2012) montrent que le pouvoir de négociation relatif des coopératives laitières aux ÉtatsUnis lorsqu'elles négocient les prix régionaux du lait à la production est faible par rapport aux entreprises de transformation du lait.

Les agriculteurs peuvent en effet négocier collectivement leur contrat par l'intermédiaire des OPs et peser ainsi dans les relations avec les entreprises pour capter une partie des gains économiques de la filière. Elles sont donc vouées à devenir des acteurs incontournables sur les marchés des produits agricoles et agroalimentaires. Le contre-pouvoir de marché des agriculteurs va dépendre du pouvoir de négociation de l'OP, mais aussi des caractéristiques du marché (localisation de la production, existence de circuits alternatifs d'approvisionnement pour les industries, capacité à contrôler l'offre totale, existence ou non de barrière à l'entrée plus ou moins forte à long terme sur le marché) qui vont influencer le prix payé aux producteurs et plus généralement les termes du contrat.

Les réglementations française et européenne ont autorisé la formation d'OPs pour rééquilibrer le pouvoir de négociation des agriculteurs vis-à-vis des industriels et de la grande distribution dans la mesure où elles respectent un certain nombre de conditions liées aux règles de concurrence ${ }^{9}$. En particulier, toute forme d'entente sur les prix est proscrite par le droit de

9. De même, aux États-Unis, le Capper-Volstead Act autorise les agriculteurs à se regrouper pour former des coopératives dont le but est de collecter, transformer et commercialiser collectivement leurs produits agricoles en accord avec la réglementation antitrust définie par le Sherman Act. 
la concurrence ${ }^{10}$. Le « paquet lait » (règlement du 14 mars 2012) porte sur les relations contractuelles dans le secteur laitier à la suite des recommandations du Groupe à Haut Niveau mobilisé par la Commission pour réfléchir au rôle de la contractualisation, du pouvoir de négociation et des organisations interprofessionnelles (Union européenne, 2012). Le règlement permet aux États membres de rendre obligatoire le recours au contrat. Il prévoit que les organisations de producteurs puissent négocier collectivement les clauses du contrat avec leurs laiteries et notamment le prix « pour toute la production de leurs membres (ou une partie de la production) dans la limite de $3,5 \%$ de la production totale de l'Union européenne et $33 \%$ de la production nationale de l'État membre ». Une réflexion sur les relations contractuelles a également été menée pour le secteur des fruits et légumes. Ces réflexions ont abouti à une modification de l'Organisation Commune de Marché (OCM) fruits et légumes en 2010 qui reconnaît maintenant les OPs et qui encourage les producteurs à se réunir en groupements de producteurs dans le but de renforcer le pouvoir de marché des agriculteurs.

\section{Efficacité économique des OPs}

La littérature en économie industrielle portant sur le pouvoir d'achat et le contrepouvoir de marché montre qu'il n'est pas forcément une bonne chose du point de vue de l'efficacité économique d'accorder un tel contre-pouvoir de marché (Dobson et Waterson, 1997 ; Chen 2003 et 2007). Si les entreprises aval ont un pouvoir de marché sur les agriculteurs, mais sont en concurrence parfaite sur le marché aval

10. Plusieurs décisions de l'Autorité de la concurrence ont sanctionné des pratiques d'entente sur les prix dans le secteur agricole, notamment dans le secteur des fraises en 2003 (03-D-36), du choufleur en 2005 (05-D-10) et des céréales en 2007 (07-D-16). (produit final de consommation), un pouvoir de marché à l'aval permet de diminuer leurs coûts d'approvisionnement en diminuant les prix de l'input agricole et cette baisse de prix d'approvisionnement sera répercutée aux consommateurs. Un contrepouvoir de marché dans ce cas aura pour effet d'augmenter les prix d'approvisionnement qui seront répercutés aux consommateurs. Par contre, si les entreprises aval ont un pouvoir de marché sur les agriculteurs, mais exercent un pouvoir de marché sur le marché aval, ce pouvoir de marché en amont combiné à celui en aval se traduit par une baisse du prix payé aux agriculteurs, mais celle-ci n'est pas répercutée aux consommateurs. Ainsi, plus les entreprises exercent un contrôle sur le marché aval et plus les effets du pouvoir de marché seront néfastes pour l'économie puisque les entreprises pourraient alors bénéficier d'un transfert de surplus provenant à la fois du producteur et des consommateurs. Dans ce cas, selon le type de contrat négocié entre les OPs et les entreprises, le résultat peut être ambigu ( $c f$. par exemple Gal-Or, 1991).

Les quelques analyses économiques sur les coopératives de négociation (bargaining cooperatives surtout présentes dans les secteurs des fruits et légumes, mais aussi du lait et de la betterave à sucre, et plutôt sur des marchés de produits transformés) aux États-Unis (Agricultural Fair Pratices Act de 1967) montrent que la mise en place d'OPs n'a pas toujours conduit à une augmentation des prix des produits agricoles aux États-Unis. Une augmentation des prix n'est possible que si la formation de l'OP permet aux producteurs de créer un réel contre-pouvoir de marché face aux industriels (Hueth et Marcoul, 2003). Ainsi, si une augmentation de prix est possible à court terme, elle est peu vraisemblable à plus long terme s'il existe une option de sortie pour les acheteurs (pas de contrôle de l'offre possible). Des prix élevés encouragent l'entrée de concurrents et 
augmentent l'offre jusqu'à ce que les prix baissent. L'OP ne peut augmenter les prix agricoles que si le nombre d'agriculteurs adhérant à l'OP est relativement important par rapport aux non-membres. Par contre, ces associations semblent avoir eu d'autres effets bénéfiques pour les agriculteurs. Les OPs peuvent en effet financer les activités promotionnelles pour l'ensemble de l'industrie, fournir des services d'assistance juridique dans un marché où les contrats comme forme de transaction sont prédominants. Elles peuvent également permettre de transmettre l'information sur les prévisions d'offre et de demande à tous les maillons de la filière en faisant le lien entre les agriculteurs et les industriels (Hueth et Marcoul, 2006).

\section{OPs et choix de la qualité}

Si les OPs jouent plusieurs rôles dont celui de contre-pouvoir de marché, elles interviennent également dans le contrôle de la qualité des produits agricoles fournis par leurs adhérents. Hendrikse et Bijman (2002) montrent comment l'hétérogénéité en matière de qualité de la production des producteurs affecte l'efficacité et la forme optimale d'une OP dans le cas où les rémunérations sont régies par un principe d'égalité. Si l'OP inclut des membres hétérogènes, les producteurs de haute qualité sont défavorisés par le principe d'égalité, mais le nombre de membres étant plus élevé, cela renforce le contre-pouvoir de marché de l'ensemble des producteurs. En revanche, si l'OP comprend des membres homogènes, les producteurs de haute qualité choisissent de constituer une OP dans le but d'exclure les producteurs de basse qualité du marché. Ils réduisent ainsi la concurrence et améliorent leur contre-pouvoir de marché.

Plusieurs reproches sont formulés à l'encontre des coopératives les qualifiant d'organisations inefficaces (Porter et Scully, 1987) parce qu'elles donnent de mauvaises incitations à leurs adhérents : les agriculteurs produisent trop et ont un comportement de passager clandestin sur la qualité. Chaque adhérent qui produit un bien agricole de qualité en supporte seul les coûts alors que les bénéfices sont partagés entre tous (Pennerstorfer et Weiss, 2013). Zago (1999) évalue l'impact d'un mode d'organisation de type coopérative en situation de monopole sur le niveau de qualité fourni sur le marché en fonction du mode de gouvernance choisi. Il révèle également l'inefficacité de ce type d'organisation en présence de producteurs hétérogènes pour produire le niveau de qualité optimal. Lorsque la majorité des adhérents est capable de produire un niveau de qualité élevée, l'OP produit en moyenne une qualité trop élevée. Inversement, si la majorité des adhérents produit un niveau de qualité basse, l'OP imposera une qualité en moyenne trop faible. Si les résultats de Zago s'appliquent dans certains pays d'Europe du Nord où des coopératives agricoles dominent certains marchés, son analyse devrait être étendue au marché oligopolistique comme cela est souvent le cas en France. De plus, pour Zago, la qualité est considérée comme un niveau d'efficacité sur la production du bien agricole. Les producteurs produisant une qualité faible sont en fait ceux pour qui la production du bien agricole est la plus coûteuse. La qualité ne se traduit pas par une augmentation du consentement à payer des consommateurs pour le produit et par conséquent n'a pas d'effet sur la demande. Enfin, Saitone et Sexton (2009) montrent que la coopérative agricole est une organisation efficace grâce à la mutualisation des revenus des adhérents, car non seulement elle fournit une assurance contre les aléas de production aux adhérents qui présentent de l'aversion au risque, mais elle limite également les incitations à produire un niveau trop élevé de qualité par rapport au niveau optimal pour la société. Cependant, selon ces auteurs, 
la coopérative est uniquement considérée comme une structure de mise en commun de la production et non pas comme une organisation avec un mode de gouvernance spécifique.

*

Depuis quelques années, les pouvoirs publics tant au niveau français qu' européen portent un intérêt particulier aux modes de coordinations horizontales (organisations de producteurs) et verticales (contrats). Ces modes de coordinations sont considérés comme des moyens possibles pour améliorer la compétitivité en améliorant la performance globale des filières agricoles, pour assurer les agriculteurs contre le risque de prix et pour assurer une redistribution de la valeur aux agriculteurs en améliorant les rapports de force au sein des filières. Dans le cas de la filière laitière par exemple, le mécanisme de quotas et de soutien au prix du lait assurait une certaine redistribution des revenus aux agriculteurs. Mais avec la suppression des quotas en 2015, la question des contrats s'est posée comme outil de redistribution et de restauration la compétitivité de la filière. Les contrats sont ainsi voués à jouer un rôle de plus en plus important dans les filières et vont avoir des répercussions fortes sur leur organisation et sur la coordination des différents opérateurs.

En nous appuyant sur la littérature, nous avons identifié dans cet article comment les contrats pouvaient répondre à ces différents objectifs et nous avons illustré leur rôle comme mécanisme d'incitation à la performance et comme mécanisme d'assurance contre les risques de prix, de production et de qualité dans le cas des filières agricoles. Les contrats, en agissant sur la coordination entre les acteurs des filières, peuvent conduire à une réduction des coûts de transaction et à une meilleure utilisation des ressources et générer ainsi des gains d'efficacité. Le recours au contrat par des groupements de producteurs peut également permettre de réduire les asymétries dans les rapports de force entre l'amont et l'aval et contrebalancer l'existence du pouvoir de marché à l'aval, améliorer le revenu des éleveurs et aider à une meilleure répartition des risques de marché dans la filière. Cependant, les contrats en résolvant certaines défaillances de marché peuvent engendrer d'autres défaillances. Par exemple, un contrat de production peut assurer les agriculteurs contre les risques de prix, mais réduire leurs incitations à la performance. De plus, les caractéristiques du marché, les stratégies de réponse des acteurs et l'existence éventuelle de politiques agricoles peuvent affecter l'efficacité des contrats à agir sur la compétitivité des filières. Ces éléments expliquent en partie le recours aux contrats en agriculture.

Cependant, nous manquons en France et plus généralement en Europe d'analyse des contrats en agriculture. Il conviendrait notamment d'analyser les modes de coordination et de contractualisation existants dans les différentes filières. Les contrats et les modes de coordination pouvant prendre différentes formes et remplir différentes fonctions, il conviendrait d'analyser à quels objectifs ces contrats répondent (/répondraient) effectivement. Une autre question est : peuvent-ils ou non résoudre les défaillances de marché identifiées cidessus et comment peuvent-ils jouer sur la structuration de la filière et contribuer à améliorer la compétitivité de cette dernière. Si ce chantier a été ouvert aux ÉtatsUnis depuis le début des années 2000, il n'existe presque rien au niveau de l'Union européenne. Or les enjeux sont importants pour les filières agricoles européennes. De plus, si les politiques publiques favorisent l'émergence d'OPs, il convient de déterminer si, effectivement, cette forme de coordination horizontale en amont de la filière doit être renforcée, dans quels cas les agriculteurs vont effectivement adhérer 
à ces formes d'organisation et quelles vont être les répercussions pour les consommateurs. Enfin, si les contrats sont plutôt développés à l'initiative des industriels et de la grande distribution, il conviendrait d'étudier plus précisément les critères d'adoption des contrats pour les agriculteurs.

\section{RÉFÉRENCES BIBLIOGRAPHIQUES}

Allain M. L., Chambolle C. (2003). Les relations entre la grande distribution et ses fournisseurs : bilan et limites de trente ans de régulation. Revue française d'économie, vol. $17, \mathrm{n}^{\circ}$ 4, pp. 169-212.

Ahn B. I., Sumner D. A. (2012). Estimation of Relative Bargaining Power in Markets for Raw Milk in the United States. Journal of Applied Economics, vol. 15, n ${ }^{\circ}$, pp. 1-23.

Azzam A. M. (1997). Measuring Market Power and Cost-Efficiency Effects of Industrial Concentration. The Journal of Industrial Economics, vol. 45, n ${ }^{\circ}$, pp. 377-386.

Baker G., Gibbons R., Murphy K. J. (2002). Relational contracts and the theory of the firm. Quaterly Journal of Economics, 117, pp. 39-83.

Bazoche P., Giraud-Héraud E., Soler L. G. (2005). Premium Private Labels, Supply Contracts, Market Segmentation and Spot Prices. Journal of Agricultural \& Food Industrial Organization, vol. $3, \mathrm{n}^{\circ} 1$.

Bijman J., Iliopoulos C., Poppe K. J., Gijselinckx C., Hagedorn K., Hanisch M., Hendrikse G. W. J., Kühl R., Ollila P., Pyykkönen P., van der Sangen G. (2012). Support for Farmers' Cooperatives. Rapport de l'Union européenne, $127 \mathrm{p}$.

Bogetoft P., Olesen H. B. (2002). Ten rules of thumb in contract design: lessons from Danish agriculture. European Review of Agricultural Economics, vol. 29, $\mathrm{n}^{\circ} 2$, pp. 185-204.

Bouamra-Mechemache Z., Yu J. (2013). Production Standards, Competition and Vertical Relationship. TSE Working Papers 13-417, Toulouse School of Economics (TSE).

Carter C. A. (2013). Futures and Options Markets. An Introduction. Rebel Text ed., 366 p.
Chen Z. (2003). Dominant retailers and the countervailing-power hypothesis. RAND Journal of Economics, vol. 34, $\mathrm{n}^{\circ} 4$, pp. 612-625.

Chen Z. (2007). Buyer Power: economic theory and antitrust policy. Research in Law and Economics $\mathrm{n}^{\circ} 22$, pp. 17-40.

Coop de France (2010). La collecte des céréales, oléagineux, et protéagineux, l'approvisionnement, la transformation.

Crespi J. M., Jones R. J. (2005). A multinomial logit framework to estimate bid shading in procurement auctions; application to cattle sales in the Texas Panhandle. Review of industrial organization, vol. $27, \mathrm{n}^{\circ} 3$, pp. 253-278.

Crespi J. M., Saitone T. L., Sexton R. J. (2012). Competition in farm product markets: do long run incentives trump short run market power? Applied Economic Perspectives and policy, vol. ${ }^{\circ} 34, \mathrm{n}^{\circ} 4$, pp. 669-695.

Danel J.-B., Malpel G.-P., Texier P.-H. (2012). Rapport sur la contractualisation dans le secteur agricole (Article L631-24 du code rural et de la pêche maritime). Conseil général de l'alimentation, de l'agriculture et des espaces ruraux, Ministère de l'Agriculture, de l'Agroalimentaire et de la Forêt.

Dedieu M. S., Courleux F. (2009). Les enjeux de la régulation du secteur laitier. MAAP, Prospective et évaluation.

Dimitri C., Jaenicke E. C., Effland A. B. (2009). Why Did Contracts Supplant the Cash Market in the Broiler Industry? An Economic Analysis Featuring Technological Innovation and Institutional Response. Journal of Agricultural \& Food Industrial Organization, vol. $7, \mathrm{n}^{\circ} 1$.

Dong F., Hennessy D. A., Jensen H. H. (2010). Contract and Exit Decisions in Finisher 
Hog Production. American Journal of Agricultural Economics, vol. 92, $\mathrm{n}^{\circ} 3$, pp. 667-684.

Dobson P. W., Waterson M. (1997). Countervailing power and consumer prices. The Economic Journal, vol. 107, $\mathrm{n}^{\circ} 441$, pp. 418-430.

Drivas K., Giannakas K. (2010). The Effect of Cooperatives on Quality-Enhancing Innovation. Journal of Agricultural Economics, vol. 61, $\mathrm{n}^{\circ} 2$, pp. 295-317.

Dubois P., Vukina T. (2004). Grower Risk Aversion and the Cost of Moral Hazard in Livestock Production Contracts. American Journal of Agricultural Economics, vol. 86, $\mathrm{n}^{\circ} 3$, pp. 835-841.

Fulponi L. (2006) Private voluntary standards in the food system: The perspective of major food retailers in OECD countries. Food Policy, $\mathrm{n}^{\circ} 31$, pp. 1-13.

Gal-Or E. (1991). Duopolistic vertical restraints. European Economic Review, vol. 35, $\mathrm{n}^{\circ}$ 6, pp. 1237-1253.

Giannakas K., Fulton M. (2005). Process innovation activity in a mixed oligopoly: The role of cooperatives. American Journal of Agricultural Economics, vol. 87, $\mathrm{n}^{\circ} 2$, pp. 406-422.

Giraud-Héraud E., Hammoudi A., Hoffmann R., Soler L.-G. (2012). Joint Private Safety Standards and Vertical Relationships in Food Retailing. Journal of Economics \& Management Strategy, vol. 21, $\mathrm{n}^{\circ} 1$, pp. 179-212.

Goodhue R. E. (2000). Broiler Production Contracts as a Multi-Agent Problem: Common Risk, Incentives and Heterogeneity. American Journal of Agricultural Economics, vol. 82, n 3, pp. 606-622.

Goodhue R. E. (2011). Food quality: The design of incentive contracts. Annual Review of Resource Economics, vol. 3, $\mathrm{n}^{\circ} 1$, pp. 119-140.

Goodhue R. E., Heien D. M., Lee H., Sumner D. A. (2003). Contracts and Quality in the CaliforniaWinegrape Industry. Review of Industrial Organization, n 23, pp. 267-282.

Goodhue R. E., Mohapatra S., Rausser G. C. (2010). Interactions Between Incentive
Instruments: Contracts and Quality in Processing Tomatoes. American Journal of Agricultural Economics, vol. 92, $\mathrm{n}^{\circ}$ 5, pp. 1283-1293.

Hardaker J., Huirne R., Anderson J., Lien G. (2004). Coping with risk in Agriculture. New York, CAB International.

Harwood J., Heifner R., Coble K., Perry J., Somwaru A. (1999). Managing Risk in Farming: Concepts, Research, and Analysis. Washington D.C., U.S. Department of Agriculture, Economic Research Service, Market and Trade Economics Division and Resource Economics Division.

Herrman R., Musser A., Weber S. (2009). The case of Germany (chapitre 4), dans Structural changes in food retailing: Ten country case studies. Food System Research Group publication, K. Stiegert et D. H. Kim.

Hendrikse G., Bijman J. (2002). On the emergence of new growers' associations: self-selection versus countervailing power. European Review of Agricultural Economics, vol. $29, \mathrm{n}^{\circ} 2$, pp. 255-269.

Hennessy D. A., Roosen J., Miranowski J. A. (2001) Leadership and the Provision of Safe Food. American Journal of Agricultural Economics, vol. 83, $\mathrm{n}^{\circ} 4$, November, pp. 862-874.

Hovelaque V., Duvaleix-Tréguer S., Cordier J. (2009). Effects of constrained supply and price contracts on agricultural cooperatives. European Journal of Operational Research, vol. 199, $\mathrm{n}^{\circ} 3$, pp. 769-780.

Hueth B., Ligon E., Wolf S., Wu S. (1999). Incentive Instruments in Fruit and Vegetable Contracts: Input Control, Monitoring, Measuring, and Price Risk. Review of Agricultural Economics, $\mathrm{n}^{\circ} 21$, pp. 374-89.

Hueth B., Marcoul P. (2003). An essay on cooperative bargaining in US agricultural markets. Journal of Agricultural \& Food Industrial Organization, vol. $1, \mathrm{n}^{\circ} 1$.

Hueth B., Marcoul P. (2006). Information sharing and oligopoly in agricultural markets: the role of the cooperative bargaining association. American Journal of Agricultural Economics, vol. 88, n $^{\circ}$ 4, pp. 866-881.

Jaenicke E. C., Shields M., Kelsey T. W. (2007). Food Processors' Use of Contracts to 
Purchase Agricultural Inputs: Evidence from a Pennsylvania Survey. Agricultural and Resource Economics Review, pp. 213-229.

Karantininis K., Sauer J., Furtan W. H. (2010). Innovation and integration in the agrifood industry. Food Policy, vol. 35, n ${ }^{\circ} 2$, pp. 112-120.

Katchova A. L., Miranda M. J. (2004). TwoStep Econometric Estimation of Farm Characteristics Affecting Marketing Contract Decisions. American Journal of Agricultural Economics, vol. 86, $\mathrm{n}^{\circ} 1$, pp. 88-102.

Key N. (2011). Does the Prevalence of Contract Hog Production Influence the Price Received by Independent Hog Producers? Journal of Agricultural and Food Industrial Organization, vol. 9, $\mathrm{n}^{\circ} 1$.

Knoeber C. R. (1989). A Real Game of Chicken: Contracts, Tournaments, and the Production of Broilers. Journal of Law, Economics, \& Organization, vol. 5, n ${ }^{\circ}$, pp. 271-292.

Lawrence J. D., Schroeder T. C., Hayenga M. L. (2001). Evolving Producer-Packer-Customer Linkages in the Beef and Pork Industries, Appl. Econ. Perspect. Pol., vol. 23, $\mathrm{n}^{\circ} 2$, pp. 370-385.

Lee H. L., Padmanabhan V., Whang S. (2004). Information distortion in a supply chain: the bullwhip effect. Management Science, 50 (12_supplement), pp. 1875-1886.

Leegomonchai P., Vukina T. (2005). Dynamic Incentives and Agent Discrimination in Broiler Production Tournaments. Journal of Economics and Management Strategy, vol. $14, n^{\circ} 4$, pp. 849-877.

Levin J. (2003). Relational Incentive Contracts. The American Economic Review, vol. 93, $\mathrm{n}^{\circ} 3$, pp. 835-857.

MacDonald J. M. (2006). Agricultural contracting, competition, and antitrust. American Journal of Agricultural Economics, vol. 88, $\mathrm{n}^{\circ} 5$, pp. 1244-1250.

MacDonald J. M., Korb P. (2011). Agricultural contracting update: Contracts in 2008. DIANE Publishing.

MacDonald J. M., Perry J., Ahearn M., Banker D., Chambers W., Dimitri C., Southard L. W. (2004). Contracts, markets, and prices: Organizing the production and use of agricultural commodities. USDA-ERS Agricultural Economic Report, $\mathrm{n}^{\circ} 837$.

Magdelaine P., Coutelet G., Riffard C. (2012). Les contrats en aviculture. Rapport de l'ITAVI pour FranceAgriMer, 59 p.

Melhim A., Shumway C. R. (2013). Cost economies in the presence of marketing contracts. Applied Economics, vol. 45, $\mathrm{n}^{\circ} 7$, pp. 921-932.

Ménard C., Klein P. G. (2004). Organizational Issues in the Agrifood Sector: Toward a Comparative Approach. American Journal of Agricultural Economics, vol. 86, $\mathrm{n}^{\circ} 3$, pp. 750-755.

Meuwissen M., Huirne R., Hardaker J. (2001). Risk and risk management: an empirical analysis of Dutch livestock farmers. Livestock Production Science, vol. 69, pp. 43-53.

Morrison Paul C. J. (2001). Cost Economies and Market Power: The Case of the U.S. Meat Packing Industry. The Review of Economics and Statistics, vol. 83, $\mathrm{n}^{\circ} 3$, pp. 531-540.

Morrison Paul C. J., Nehring R. Banker D. (2004). Productivity, Economies, and Efficiency in U.S. Agriculture: A Look at Contracts. American Journal of Agricultural Economics, vol. 86, $\mathrm{n}^{\circ}$ 5, pp. 1308-1314.

OECD (2006). Supermarkets and the Meat Supply Chain: The Economic Impact of Food Retail on Farmers, Processors and Consumers. OECD Publishing. doi: 10.1787/9789264028883-en.

Patrick G. F., Peiter A. J., Knight T. O., Coble T. K., Baquet A. (2007). Hog Producers' Risk Management Attitudes and Desire for Additional Risk Management Education. Journal of Agricultural and Applied Economics, vol. 39, $\mathrm{n}^{\circ}$ 3, pp. 671-687.

Paulson N. D., Katchova A. L., Lence S. H. (2010). An empirical analysis of the determinants of marketing contract structures for corn and soybeans. Journal of Agricultural \& Food Industrial Organization, vol. 8, $\mathrm{n}^{\circ} 1$.

Pennerstorfer D., Weiss C. R. (2013). Product quality in the agri-food chain: do cooperatives offer high-quality wine? European Review of Agricultural Economics, vol. 40, $\mathrm{n}^{\circ}$ 1, pp. 143-162.

Pennings J. M. E., Garcia P. (2001). Measuring Producers' Risk Preferences: A Global 
Risk-Attitude Construct. American Journal of Agricultural Economics, vol. 83, $\mathrm{n}^{\circ} 4$, pp. 993-1009.

Pennings J. M., Leuthold R. M. (2000). The role of farmers' behavioral attitudes and heterogeneity in futures contracts usage. American Journal of Agricultural Economics, vol. 82, n 4, pp. 908-919.

Pietola K., Wang H. (2000). The value of priceand quantity-fixing contracts for piglets in Finland. European Review of Agricultural Economics, vol. 27, n 4, pp. 431-447.

Porter P. K., Scully G. W. (1987). Economic Efficiency in Cooperatives. Journal of Law and Economics, vol. 30, $\mathrm{n}^{\circ}$ 2, pp. 489-512.

Reimer J. J. (2006). Vertical integration in the pork industry. American Journal of Agricultural Economics, vol. 88, $\mathrm{n}^{\circ} 1$, pp. 234-248.

Reynaud A., Ricome A. (2010). An Empirical Analysis of the Determinants of Marketing Contract Choices in France, Journées INRA SFER CIRAD, décembre.

Roe B., Sporleder T. L., Belleville B. (2004). Hog Producer Preferences for Marketing Contract Attributes. American Journal of Agricultural Economics, vol. 86, $\mathrm{n}^{\circ} 1$, pp. 115-123.

Roguet C., Rieu M. (2011). Les groupements de producteurs de porcs en France : une organisation originale. Communication présentée aux $11^{\text {es }}$ Journées Productions porcines et avicoles.

Saitone T. L., Sexton R. J. (2009). Optimal Cooperative Pooling in a Quality-Differentiated Market. American Journal of Agricultural Economics, vol. 91, $\mathrm{n}^{\circ} 5$, pp. 1224-1232.

Salanié B. (2005). The Economics of Contracts: A Primer. MIT Press, second edition.

Sexton R. J. (2013). Market power, misconceptions, and modern agricultural markets. American Journal of Agricultural Economics, vol. 95, n² 2, pp. 209-219.

Sexton R. J., Lavoie N. (2001). Food processing and distribution: an industrial organization approach. Handbook of agricultural economics, $\mathrm{n}^{\circ} 1$, pp. 863-932.

Trienekens J., Zuurbier P. (2008). Quality and safety standards in the food industry, developments and challenges. International Journal of Production Economics vol. 113, $\mathrm{n}^{\circ} 1$, pp. 107-122.

Union européenne (2012). Règlement $n^{\circ}$ 261/2012 du Parlement européen et du Conseil du 14 mars 2012 portant modification du règlement (CE) $n^{\circ} 1234 / 2007 d u$ Conseil en ce qui concerne les relations contractuelles dans le secteur du lait et des produits laitiers. http://eur-lex.europa.eu/ LexUriServ/LexUriServ.do?uri=OJ:L:2012: 094:0038:0048:FR:PDF.

Valceschini E. Saulais L. (2005). Articulation entre réglementation, normalisation et référentiels privés dans les industries agroalimentaires. Rapport pour le ministère de l'Agriculture et de la Pêche. 117 p.

Vavra P. (2009). Role, Usage and Motivation for Contracting in Agriculture ( $\mathrm{n}^{\circ}$ 16). OECD Publishing.

Vukina T. Leegomonchai P. (2006). Oligopsony power, asset specificity and hold-up: evidence from the broiler industry. American Journal of Agricultural Economics, vol. 88, $\mathrm{n}^{\circ} 3$, pp. 589-605.

Ward C. E., Koontz S. R., Schroeder T. C. (1998). Impacts from captive supplies on fed cattle transaction prices. Journal of Agricultural and Resource Economics, pp. 494-514.

Ward C. E. (2002). A review of causes for and consequences of economic concentration in the US meatpacking industry. Current Agriculture, Food \& Resource Issues, $\mathrm{n}^{\circ} 3$, pp. 1-28.

Williamson O. (1975). Markets and Hierarchies: Analysis and Antitrust Implications. New York, Free Press.

Wolf C. A. (2012). Dairy farmer use of price risk management tools. Journal of Dairy Science, vol. 95, $\mathrm{n}^{\circ}$ 7, pp. 4176-4183.

Wu S. Y., Roe B. (2007). Contract Enforcement, Social Efficiency, and Distribution: Some Experimental Evidence. American Journal of Agricultural Economics, vol. 89, $\mathrm{n}^{\circ} 2$, pp. 243-258.

Xia T. Sexton R. (2004). The competitive implication of top of the market and related contract-pricing clauses. American Journal of Agricultural Economics, vol. 86, $\mathrm{n}^{\circ} 1$, pp. 124-138. 
Zago A. M. (1999). Quality and self-regulation in agricultural markets: how do Producer Organisations make the rules? European Review of Agricultural Economics, vol. 26, $\mathrm{n}^{\circ} 2$, pp. 199-218.

Zhang T. Brorsen B. W. (2010). The long run and short run impact of captive supplies on the spot market price: an agentbased artificial market. American Journal of Agricultural Economics, vol. 92, $\mathrm{n}^{\circ} 4$, pp. 1181-1194.

Zheng X. Vukina T. (2007). Efficiency gains from organizational innovation: Comparing ordinal and cardinal tournament games in broiler contracts. International Journal of Industrial Organization, $\mathrm{n}^{\circ}$ 25, pp. 843-859.

Zheng X., Vukina T., Shin C. (2008). The role of farmers' risk aversion for contract choice in the US hog industry. Journal of Agricultural \& Food Industrial Organization, vol. 6, $\mathrm{n}^{\circ} 1$.

Zeuli K. A., King R. P. (2004). The impact of organizational form on producer contracting decisions. Canadian Journal of Agricultural Economics/Revue canadienne d'agroéconomie, vol. 52, $\mathrm{n}^{\circ}$ 2, pp. 147-164. 